\title{
SPECTROSCOPY OF THE GLOBULAR CLUSTERS IN M87
}

\author{
J.R. Mould, J.B. Oke, and J.M. Nemec \\ Palomar Observatory, Caltech 105-24 \\ Pasadena CA 91125 \\ U.S.A.
}

\begin{abstract}
With a velocity dispersion of $370 \pm 50 \mathrm{~km} / \mathrm{sec}$ the globular cluster system of M87 is kinematically hotter than the stars in the giant elliptical itself. This is consistent with the clusters' shallower density distribution for isotropic orbits. The mean metallicity of the 27 clusters in the sample analyzed here is no more than a factor of 2 more metal rich than the cluster system of the Milky Way, but considerably more metal poor than the integrated starlight in the field at a radius of $1^{\prime}$ from the center of M87. There is no evidence for the existence of young clusters in the system. The mass-radius relation between $1^{\prime}$ and $5^{\prime}$ required to contain the globular clusters joins on to that required to contain the hot gas around M87.
\end{abstract}

We have obtained multi-slit spectra of a total of 52 objects from Strom et al (1981) lying within $\mathbf{7}^{\prime}$ of the center of M87. The principal dynamical conclusions are:

1. Twenty-seven of the objects are globular clusters with a mean heliocentric velocity of $1320 \mathrm{~km} / \mathrm{sec}$ and a dispersion of $370 \pm 50 \mathrm{~km} / \mathrm{sec}$.

2. The cluster data do not fit an extension of the $\mathrm{M} / \mathrm{L}=$ constant model of Sargent et al (1978) (see Figure 1). They are consistent with $\sigma(\mathrm{r})=$ constant. But the sample size must be doubled in order to make a definite statement on the presence of dark matter in ellipticals.

3. If the velocity dispersion $\sigma(\mathrm{r})$ is constant, $\sigma_{\text {globs }} / \sigma_{\text {stars }}=1.4 \pm 0.2$ at $1^{\prime}$, For isotropic orbits this is consistent with the greater radial concentration of the starlight (see Harris 1986).

4. With $\sigma(\mathbf{r})=$ constant, isotropic orbits and a cluster volume density fall off like $r^{-2.1}$,

$$
\begin{aligned}
\mathrm{M}(\mathrm{r}) & =6 \times 10^{10} \mathrm{r}(\mathrm{kpc}) M_{\odot} \\
& =3 \times 10^{11} \mathrm{r}(\mathrm{arcmin}) M_{\odot} \text { for } \mathrm{M} 87 \text { at } 15 \mathrm{Mpc} .
\end{aligned}
$$

This mass-radius relation makes the connection between previous optical studies and that inferred from the $\mathrm{x}$-ray gas around M87 by Fabricant and Gorenstein (1983).

We gratefully acknowledge the partial support of this project by NASA grant NGL-05-002-134 and NSF grant AST-8502518. 


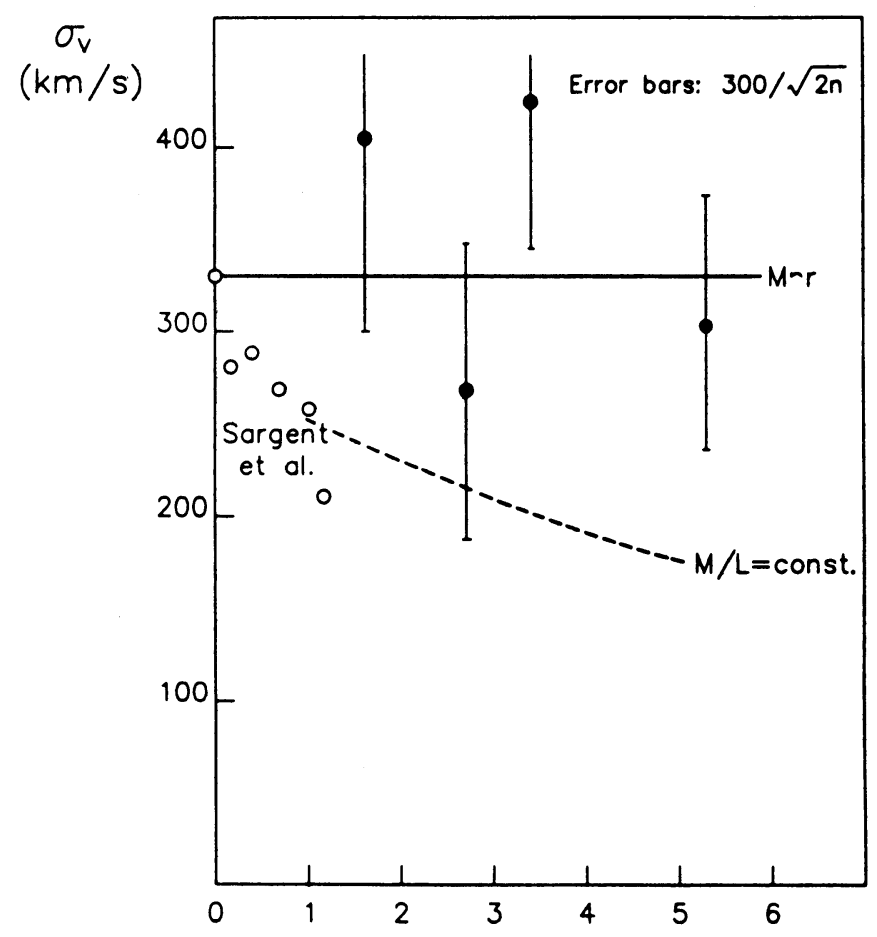

Figure 1. Velocity dispersion profile in 4 radial bins. The abscissa is labelled in minutes of arc.

\section{REFERENCES}

Fabricant, D, \& Gorenstein, P., 1983. Astrophys. J., 267, 535.

Harris, W., 1986. Astron. J., 91, 822.

Sargent, W., Young, P., Boksenberg, A., Shortridge, K., Lynds, C.R., \& Hartwick, F.D.A., 1978. Astrophys. J., 221, 731.

Strom, S., Forte, J.C., Harris, W., Strom, K., Wells, D., \& Smith, M., 1981. Astrophys. J., 245, 416. 